\title{
ABORDAGEM FISIOTERAPÊUTICA NO CENTRO MULTIDISCIPLINAR DE DOR OROFACIAL DA UFSC: UM RELATO DE EXPERIÊNCIA
}

\author{
Cleide Mara Niszezak \\ Universidade Federal de Santa Catarina \\ cleniszezak@gmail.com \\ Maynara Schlickmann de Freitas \\ Universidade Federal de Santa Catarina \\ maynarafreitas@hotmail.com \\ Luiza Pereira do Nascimento \\ Universidade Federal de Santa Catarina \\ luizanascimento27@gmail.com
}

\author{
Morgane Marion Kuntze \\ Universidade Federal de Santa Catarina \\ kuntze.morgane@gmail.com
}

Fernanda Berretta

Universidade Federal de Santa Catarina feberretta@hotmail.com

Beatriz Dulcinéia Mendes de Souza Universidade Federal de Santa Catarina dentbia@gmail.com

André Luís Porporatti Universidade Federal de Santa Catarina andreporporatti@yahoo.com.br

Resumo

O objetivo deste trabalho é descrever as atividades fisioterapêuticas desenvolvidas no Centro Multidisciplinar de Dor Orofacial (CEMDOR) do curso de Odontologia da UFSC no período de março a julho de 2018. O CEMDOR realiza diagnóstico, tratamento e acompanhamento de pacientes com Disfunções Temporomandibulares (DTM), Distúrbios do Sono e Dor Orofacial por meio da integração de diversos profissionais da área da saúde. Junto aos alunos de Odontologia, os fisioterapeutas participam das anamneses e planejamentos dos casos, além de realizarem tratamentos específicos da área. Esses tratamentos baseiam-se em exercícios, liberações miofasciais e de pontos gatilho, orientações domiciliares, alongamentos, técnicas de mobilização articular, osteopatia, reeducação postural e respiratória, entre outras. Durante os tratamentos, pode-se evidenciar melhora em relação à amplitude de movimento, do quadro de dor, além da recuperação da função e fortalecimento do sistema musculoesquelético. Esses achados demonstram que a fisioterapia tem papel fundamental na melhora e controle das DTMs e dores orofaciais, melhorando a qualidade de vida dos pacientes.

Palavras-chave: Disfunção Temporomandibular. Dor Orofacial. Odontologia. Fisioterapia.

\section{PHYSIOTHERAPEUTIC APPROACH IN TEMPOROMANDIBULAR DISORDERS IN CEMDOR UFSC: AN EXPERIENCE REPORT}

Abstract

The aim of this work is to describe the physiotherapeutic activities developed at the Multidisciplinary Orofacial Pain Center (CEMDOR) of Dentistry at UFSC in the period from march to July of 2018. CEMDOR performs diagnosis, treatment and follow-up of patients with Temporomandibular Disorders (TMDs), Sleep Disorders and Orofacial Pain through the integration of several health areas. Together with the Dentistry students, physiotherapists participate in the anamneses and case studies, besides performing specific treatments of the area. These treatments are based on exercises, myofascial releases and trigger points, home guidelines, stretching, joint mobilization techniques, osteopathy, postural and respiratory reeducation, among others. During the treatments, it was possible to show improvement in the range of motion, pain, function recovery and strengthening of the musculoskeletal system. These findings demonstrate that physiotherapy plays a fundamental role in the improvement and control of TMDs and orofacial pain, improving patients' quality of life.

Keywords: Temporomandibular Disorder. Orofacial Pain. Dentistry. Physiotherapy.

\section{ENFOQUE FISIOTERAPÉUTICO EN LAS DISFUNCIONES TEMPOROMANDIBULARES EN EL CEMDOR UFSC: UN RELATO DE EXPERIENCIA}

Resumen

El objetivo de este trabajo es describir las actividades fisioterapéuticas desarrolladas en el Centro Multidisciplinar de Dolor Orofacial (CEMDOR) en la UFSC, en el período de marzo a julio de 2018. CEMDOR realiza diagnóstico, tratamiento y acompañamiento de pacientes con Disfunciones Temporomandibulares (DTM), Disturbios del Sueño y Dolor Orofacial por medio de la integración de diversas áreas de la salud. Fisioterapeutas participan de las anamnesis y planificaciones de los casos, además de realizar tratamientos específicos del área. Estos tratamientos se basan en ejercicios, liberaciones miofasciales y puntos gatillo, orientaciones domiciliares, estiramientos, técnicas de movilización articular, osteopatía, reeducación postural y respiratoria, entre otras. Durante los tratamientos se pudo evidenciar mejoría en relación a la amplitud de movimiento, del cuadro álgico, además de la recuperación de la función y fortalecimiento del sistema musculoesquelético. Estos hallazgos demuestran que la fisioterapia tiene un papel fundamental en la mejora y control de las DTM y los dolores orofaciales, mejorando la calidad de vida de los pacientes.

Palavras clave: Disfunción Temporomandibular. Dolor Orofacial. Odontología. Fisioterapia. 
Abordagem fisioterapêutica no Centro Multidisciplinar de Dor Orofacial da UFSC: um relato de experiência

\section{INTRODUÇÃO}

O termo disfunção tempomandibular (DTM) compreende uma série de alterações funcionais que atingem os músculos da mastigação, bem como a articulação temporomandibular (ATM) e estruturas orofaciais associadas ( DE LEEUW; KLASSER 2013). A DTM é um problema de saúde pública significativo, pois afeta uma elevada proporção da população. É a segunda condição musculoesquelética que mais afeta a população, seguida após dor lombar crônica, resultando em dor e incapacidade ao indivíduo (SHIFFMAN et al, 2014; SIERWALD et al, 2015).

A prevalência da DTM tem sido relatada entre 3,7\% a $12 \%$, com impacto significativo em fatores físicos e psicossociais, sendo três a cinco vezes mais frequente em mulheres (MARTÍNEZ et al, 2018) que homens. A DTM tende a ocorrer após a puberdade, com a maior prevalência ocorrendo em mulheres de 20 a 40 anos. A sua distribuição por sexo e idade sugere uma possível ligação entre sua patogênese e o eixo hormonal feminino. Estudos sugerem que os hormônios sexuais podem predispor à disfunção da ATM (CUCCIA et al., 2011).

As DTMs possuem etiologia multifatorial e estão relacionadas à fatores estruturais, neuromusculares, psicológicos (ansiedade, estresse), bruxismo (apertar, bater ou ranger os dentes durante o sono e apertar os dentes em vigília) (DONARUMMA et al., 2010; ROSSI et al., 2014), hábitos parafuncionais (roer unhas, morder objetos, mascar chicletes, morder o lábio ou bochechas), lesões traumáticas ou ainda degenerativas da ATM (CUCCIA et al., 2011; DONARUMMA et al., 2010; REZAZADEH et al., 2016). A Academia Americana de Dor Orofacial estima que 40 a 75\% da população apresente pelo menos um sinal da doença e relata que 33\% da população tem pelo menos um sintoma (CUCCIA et al., 2011). Considerando que a proporção de influências psicoemocionais está cada dia mais elevada, pode-se esperar que o número de pacientes acometidos por alguma DTM aumente nos próximos anos (HALMOVA et al, 2017).

As DTM podem ser classificadas como musculares ou articulares. As articulares correspondem a alterações no funcionamento do complexo côndilo-disco (deslocamento do disco com e sem redução), artralgias inflamatórias e não inflamatórias, desordens de hipomobilidade (adesão, anquilose/aderência) e desordens de hipermobilidade (subluxação). As DTMs musculares por sua vez podem ser classificadas em contraturas, mialgias, dores miofasciais (com espalhamento ou com referência) e espasmos (SHIFFMAN et al, 2014).

Os sintomas mais frequentes das DTM referem-se à dor inespecífica na região temporomandibular, amplitude de movimento limitada, e ruídos articulares (RAYA et al., 2017; 
Abordagem fisioterapêutica no Centro Multidisciplinar de Dor Orofacial da UFSC: um relato de experiência

HALMOVA et al., 2017), além de sensibilidade muscular, cefaleia , zumbido, dor orofacial, dor durante a mastigação (REZAZADEH et al., 2016; DONARUMMA et al., 2010), dores irradiadas para os ouvidos, dores no pescoço e/ou rigidez, sensação de plenitude auricular, tonturas e vertigens. A adição ou a exacerbação desses sinais e sintomas pode limitar ou mesmo incapacitar o indivíduo em suas atividades funcionais(CUCCIA et al., 2011; DONARUMMA et al., 2010; ROSSI et al., 2014). A dor da DTM tem um impacto negativo na qualidade de vida do paciente, prejudicando as atividades de trabalho, da escola, o sono e o apetite/alimentação, além de estar associada a comorbidades, como depressão e outros fatores psicológicos (DONNARUMA et al., 2010; MARTÍNEZ et al, 2018).

O diagnóstico precoce da DTM é fundamental para prevenir a deterioração e formação da dor crônica, com todas as suas consequências psicossociais e funcionais (HALMOVA et al, 2017). O mesmo se baseia no exame clínico, na história da doença e em outros métodos, como questionários de pesquisa. Além disso o diagnóstico deve incluir uma inspeção palpação cuidadosa de músculos e da ATM (REZAZADEH et al., 2016).

O tratamento das DTMs envolve uma equipe multidisciplinar, com o objetivo de promover qualidade de vida ao paciente. O CEMDOR é um projeto inserido no curso de graduação de Odontologia, vinculado à Universidade Federal de Santa Catarina-UFSC, voltado a extensão universitária por meio de atendimento gratuito à comunidade, sendo um centro de referência em diagnóstico, tratamento e acompanhamento de pacientes com DTM, Distúrbios do Sono e Dor Orofacial. As intervenções realizadas dentro do projeto, contam com alunos e profissionais da Odontologia, Especialistas em Sono, Acupuntura, Auriculoterapia, Homeopatia, especialistas em Hipnose terapêutica, Fonoaudiólogos, Médicos Residentes do Hospital Universitário (HU) da UFSC em dor e Fisioterapeutas. Esta atuação em equipe busca identificar as possíveis origens das disfunções, permitindo ações terapêuticas que não se limitam à sintomatologia, mas identificam e tratam os fatores causadores, prevenindo assim as recidivas.

O tratamento fisioterapêutico aplicado no projeto se baseia, de uma forma geral, em exercícios, liberações miofasciais, liberações de pontos gatilho de dor, termoterapia, alongamentos, técnicas de mobilização articular, osteopatia, reeducação postural, reeducação respiratória, acompanhamento e orientações gerais aos pacientes.

De acordo com a literatura, a fisioterapia mostra-se efetiva na redução da dor muscular, aumento da amplitude de movimento, reeducação postural, diminuição da inflamação e redução da sobrecarga muscular na ATM, recuperando função e fortalecendo o sistema musculoesquelético (TENREIRO, SANTOS, 2018). 
Abordagem fisioterapêutica no Centro Multidisciplinar de Dor Orofacial da UFSC: um relato de experiência

Neste contexto, tendo em vista a complexidade dos mecanismos envolvidos na Dor Orofacial e na DTM, é de grande relevância uma abordagem multidisciplinar, visando sobretudo a melhora dos sintomas dolorosos e funcionais, os quais influenciam na qualidade de vida dos pacientes. Desta forma, o objetivo deste trabalho é descrever as atividades desenvolvidas e as técnicas fisioterapêuticas utilizadas durante os atendimentos a pacientes do CEMDOR na UFSC.

\section{MATERIAIS E MÉTODOS}

A atuação da Fisioterapia em parceria com o CEMDOR (UFSC), iniciou-se no primeiro semestre de 2018 e busca o atendimento de pacientes previamente cadastrados de forma online. As avaliações e tratamentos são disponibilizadas gratuitamente nas segundas-feiras no período vespertino.

Inicialmente os pacientes são acolhidos e avaliados na clínica de Odontologia da UFSC, por alunos da nona fase sob a forma de estágio supervisionado, os quais recebem auxílio de alunos da pós-graduação (mestrandos e doutorandos em Odontologia) sob a coordenação dos Professores André Porporatti e Beatriz D. M. Souza. Uma avaliação completa composta por anamnese e exame clínico detalhado em relação à DTM é realizada, seguida de elaboração diagnóstica e implementação do tratamento mais adequado. $\mathrm{O}$ tratamento pode incluir confecção de placas oclusais, aparelhos de avanço mandibular para ronco e apneia, agulhamento seco, viscossuplementação da ATM com hialuronato de sódio, prescrição farmacológica, acupuntura, homeopatia, hipnose terapêutica, tratamento fonoaudiológico e tratamento fisioterapêutico.

$\mathrm{Na}$ maioria dos casos, há encaminhamento para a equipe de fisioterapia. Os atendimentos são realizados na própria clínica do CEMDOR. O tratamento visa proporcionar melhora das dores, recuperação da função, conscientização dos pacientes em relação à disfunção, orientações sobre o tratamento e sobre hábitos da vida diária.

As técnicas específicas da fisioterapia para o tratamento das DTMs incluem: terapia manual com pompagens na musculatura da ATM e região cervical; liberação de pontos gatilho intra e extraoral e na região suboccipital; liberação miofascial; técnicas de mobilização articular passiva (distração articular); exercícios ativos/passivos/resistidos; termoterapia; alongamentos; técnicas de osteopatia cervical, craniana e da ATM; exercícios de correção postural; reeducação respiratória; orientação sobre a correção de hábitos parafuncionais.

Para mensuração da amplitude de movimento articular da ATM foi utilizado exame físico de palpação, evidenciando diferenças na mobilidade dos côndilos antes e após o uso das técnicas. Para mensuração da amplitude de movimento mandibular de abertura (mm), foi utilizado um 
Abordagem fisioterapêutica no Centro Multidisciplinar de Dor Orofacial da UFSC: um relato de experiência

paquímetro para confirmação dos dados. Além disso os pacientes foram questionados subjetivamente sobre os efeitos das técnicas após cada sessão de fisioterapia e nas semanas subsequentes.

\section{RESULTADOS E ANÁLISES}

Existem, como já descritas, inúmeras técnicas fisioterápicas específicas e que são aplicadas no atendimento aos pacientes do CEMDOR. A terapia manual influencia na redução da dor orofacial devido ao relaxamento muscular, à redução da hiperatividade muscular e ao ganho de mobilidade articular, promovendo restabelecimento da função muscular normal (OLIVO et al, 2015). Fisiologicamente, sugere-se que a analgesia imediata induzida manualmente pode estar relacionada a um bloqueio sensorial, com a diminuição dos impulsos nervosos periféricos para o sistema nervoso central (SNC), contribuindo para o alívio da dor (FERREIRA et al, 2016).

$\mathrm{Na}$ liberação de pontos gatilho na musculatura de ATM e região suboccipital é realizada por meio de pressão isquêmica. Esta consiste numa compressão local com intensidade controlada, provocando melhora no aporte sanguíneo e de oxigênio, estimulando a propriocepção, a liberação de aderências fibrosas e a produção de fluido sinovial (OLIVO et al, 2015; TENREIRO; SANTOS, 2018).

A termoterapia é uma recomendação para o tratamento domiciliar. Muitos pacientes relatam alívio da dor após o uso de compressas de calor ou gelo na região dolorosa. A aplicação local de calor pode aumentar a circulação e relaxar os músculos, enquanto o gelo pode servir no tratamento de processos inflamatórios musculares e articulares. Não obstante, os alongamentos também são recomendados e podem ser executados de 3 a 4 vezes ao dia para maximizar eficácia do tratamento (ROSSI et al., 2014).

A redução da dor e o aumento da amplitude do movimento articular na ATM, pode ser alcançada por meio da mobilização articular passiva com técnicas de distração articular da ATM (TENREIRO, SANTOS, 2018; HALMOVA et al., 2017).

Olivo et al. (2015) descrevem que a mobilização manual da coluna cervical também é efetiva para tratar pacientes com dor cervico-craniofacial de origem miofascial devido a redução da intensidade e da sensibilidade à dor nessas regiões.

A intervenção através de exercícios ativos/passivos/resistidos da musculatura mastigatória e da região cervical tem o objetivo de melhorar força e mobilidade da região (HALMOVA et al, 2017). Exercícios isométricos da musculatura facial - alternadamente contra a resistência oposta à abertura, ao fechamento, à excursão lateral e à protrusão - aumentam o fluxo 
Abordagem fisioterapêutica no Centro Multidisciplinar de Dor Orofacial da UFSC: um relato de experiência

sanguíneo dos músculos e a consciência corporal do paciente em relação à musculatura (FERREIRA et al, 2016).

Os exercícios de reeducação postural no tratamento das DTMs destacam que a posição corporal incorreta em combinação com a compressão da raiz espinhal e a contração isométrica prolongada dos músculos, além de outras alterações degenerativas nos tendões e ligamentos, são os fatores etiológicos mais importantes do distúrbio (HALMOVA et al., 2017). A efetividade dos exercícios de reeducação/correção postural é reconhecida, uma vez que estudos comprovaram que pacientes tratados com treinamento postural têm significativamente menos dor e menos recidivas de DTM (OLIVO et al., 2015).

Um estudo de revisão de literatura realizado por Ferreira (2015), acrescenta um importante recurso fisioterapêutico, a osteopatia. Trata-se de uma abordagem terapêutica que visa corrigir ou minimizar os efeitos nocivos ao corpo humano dadas pela disfunção somática. É utilizado para tratar problemas biomecânicos por meio de um conjunto de manobras específicas (que podem ser articulares, musculares ou fasciais), sendo uma alternativa para o tratamento das DTMs e dores orofaciais. Portanto, o tratamento osteopático na região cervical de $\mathrm{CO} / \mathrm{C} 1 / \mathrm{C} 2 / \mathrm{C} 3$ poderá influenciar na resposta homeostática corporal dos indivíduos portadores de DTM. Em geral, os pacientes relatam que os resultados das intervenções osteopáticas são satisfatórias quanto ao alívio da dor e melhora da mobilidade articular cervical.

Quanto aos hábitos parafuncionais, destaca-se a tentativa de conscientizar o paciente quanto ao apertamento dentário em vigília (através da sugestão de uso do aplicativos "Desencoste" ou pequenos adesivos que executem essa função), não mascar chicletes, não morder os lábios, não apoiar o queixo sobre a mão, não roer unhas e objetos (ROSSI et al., 2014).

Por conseguinte, a reeducação respiratória utiliza-se da prática dos padrões respiratórios diafragmático, intercostal e apical, e tem como objetivo a conscientização do paciente sobre seus movimentos respiratórios e a melhora da oxigenação tecidual (PINHEIRO et al., 2006).

Embora todas essas terapias sejam preconizadas na literatura por diversos autores, para que haja fluidez e sucesso nos tratamentos, são imprescindíveis a conscientização e a cooperação do paciente quanto ao tratamento e às mudanças de hábitos comportamentais, afim de manter os ganhos obtidos na terapia.

Todas as atividades fisioterapêuticas descritas anteriormente foram realizadas em pacientes com DTM e/ou dor orofacial mostraram-se eficazes na maioria das queixas relatadas pelos pacientes. Durante os tratamentos pôde-se evidenciar a melhora significativa em relação à dor, amplitude de movimento articular na ATM e na amplitude mandibular de abertura. Um 
Abordagem fisioterapêutica no Centro Multidisciplinar de Dor Orofacial da UFSC: um relato de experiência

aumento na lubrificação articular foi evidenciado pela redução dos cliques articulares, além da redução das dores musculares e articulares.

Tais resultados demonstram o papel essencial do tratamento fisioterapêutico na qualidade de vida dos pacientes que sofrem com dor orofacial e DTM.

O acompanhamento dos pacientes durante a evolução do tratamento demonstrou resultados positivos no quadro doloroso e na funcionalidade da ATM e melhora na qualidade de vida. Além disso, percebeu-se relevante engajamento dos mesmos nas mudanças de hábitos diários e nas correções posturais propostas.

A Fisioterapia desempenha um papel adjuvante em praticamente todos os regimes de tratamento de DTM, de modo que é comumente utilizada para aliviar os distúrbios musculoesqueléticos relacionados à dor, contraturas musculares e processos inflamatórios, possibilitando a restauração da função motora oral.

Percebe-se, contudo, a importância de um tratamento multidisciplinar adequado e a elaboração de informações que capacitem o paciente a realizar o autocuidado, sobretudo gerenciando seus sintomas e complementando o tratamento em âmbito domiciliar.

Após esta experiência, percebemos a importância de quantificar as medidas em futuros estudos, possibilitando o desenvolvimento de protocolos de atendimento eficazes para o tratamento da DTM.

\section{CONSIDERAÇÕES FINAIS}

Em meio à crescente demanda de pacientes com DTM, surge a necessidade de um atendimento multidisciplinar na área da saúde, integrando áreas como fisioterapia, odontologia, medicina, psicologia e fonoaudiologia. Para uma correta indicação terapêutica, a avaliação dos possíveis sintomas juntamente com o trabalho em equipe é fundamental.

Neste contexto, a fisioterapia tem promovido melhora na qualidade de vida dos indivíduos com DTM. Foi observado no período de acompanhamento melhora funcional na ATM e alívio do quadro doloroso após a aplicação das técnicas fisioterapêuticas, como: a terapia manual; liberação de pontos gatilho e miofascial; mobilização articular passiva; exercícios ativos/passivos/resistidos; termoterapia; alongamentos; osteopatia; exercícios de correção postural; reeducação respiratória e a correção de hábitos parafuncionais

O tratamento baseado em um acolhimento humanizado ao paciente e a colaboração deste, são imprescindíveis para o sucesso do mesmo. Desta forma, os objetivos são alcançados 
Abordagem fisioterapêutica no Centro Multidisciplinar de Dor Orofacial da UFSC: um relato de experiência

mais facilmente, fornecendo aos pacientes melhora significativa da qualidade de vida e, aos profissionais, importantes conhecimentos práticos.

\section{REFERÊNCIAS}

CUCCIA, A. M. et al. Manual Therapy of the Mandibular Accessory Ligaments for the Management of Temporomandibular Joint Disorders. The Journal of the American Osteopathic Association, v. 111, n. 2, p. 102-112, fev 2011.

DE LEEUW, R.; KLASSER, G. D. Diagnosis and management of TMDs. Orofacial pain guidelines for assessment, diagnosis, and management. 5th ed. USA: Quintessence Publishing Co, Inc, p. 127-186, 2013.

DONNARUMA, M. D. C. et al. Disfunções Temporomandibulares: Sinais, Sintomas e Abordagem Multidisciplinar. Revista Cefac São Paulo, v. 12, n. 5, Set/Out. 2010.

FERREIRA, L. T. Osteopatia como meio Terapêutico nas Disfunções Temporomandibulares e Dores Orofaciais. Dissertação de mestrado, UNESP, 2015.

FERREIRA et al. Abordagem Fisioterapêutica no Tratamento da Disfunção Temporomandibular. Revista Inspirar Saúde \&Movimento, ed. 38, v.9, 2016.

MARTÍNEZ, A. G. et al. Management of pain in patients with temporomandibular disorder (TMD): challenges and solutions. Journal of Pain Research, v.11, p. 571-587, 2018.

HALMOVA, K. et al. The influence of cranio-cervical rehabilitation in patients with myofascial temporomandibular pain disorders. Bratislava Medical Journal, vol.118, n,11. p.710-713, 2017.

OLIVO, S. A. et al. Effectiveness of Manual Therapy and Therapeutic Exercise for Temporomandibular Disorders: Systematic Review and Meta-Analysis. Journal of the American Physical Therapy Association, v. 96, n. 1, aug, 2015.

PINHEIRO, C. H. J, E., et al. Modificação do Padrão Respiratório Melhora o Controle Cardiovascular. Sociedade Brasileira de Cardiologia, ed. 88, v. 6,p. 651-659, 2006.

RAYA, C. R. et al. Role of upper cervical spine in temporomandibular disorders.Journal of Back and Musculoskeletal Rehabilitation, p. 1-6, nov 2017.

REZAZADEH, F. et al. Comparison of the Effects of Transcutaneous Electrical Nerve Stimulation and Low-Level Laser Therapy on Drug-Resistant Temporomandibular Disorders. Journal of Dentistry of Shiraz University of Medical Sciences, v.18, n. 3, p.187-192, september, 2016.

ROSSI, S. S. et al. Temporomandibular Disorders: Evaluationand Management. Medical Clinics of North America, v. 98, p. 1353-1384, nov, 2014.

SHIFFMAN, E. et al. Diagnostic Criteria for Temporomandibular Disorders (DC/TMD) for Clinical and Research Applications. Journal of Oral Facial Pain and Headache, v.28, n.1, p.627, 2014. 
Abordagem fisioterapêutica no Centro Multidisciplinar de Dor Orofacial da UFSC: um relato de experiência

SIERWALD, I. et al. Association of temporomandibular disorder pain with awake and sleepe bruxism in adults. Journal of Orofacial Orthopedics/ Fortschritte der Kieferorthopadie, v.76,n.4,p.305-317, Jul 2015.

TENREIRO, M.J.S; SANTOS, R. Terapia Manual nas Disfunções da ATM. 2.ed. Rio de Janeiro: Ed. Rubio. 2018.

Recebido em: 05/09/2018

Aceito em: 02/04/2019 